\title{
An UWB Bandpass Filter with Large Notch Suppression
}

\author{
Ruibing Dong, Ramesh K. Pokharel, Haruichi Kanaya, and Keiji Yoshida, \\ Graduate School of ISEE, Kyushu University \\ Motooka 744, Nishi-ku, Fukuoka 819-095, Japan \\ dong@yossvr3.ed.kyushu-u.ac.jp
}

\begin{abstract}
We present an ultra-wideband bandpass filter with $-15 \mathrm{~dB}$ suppression between $5.15-5.8 \mathrm{GHz}$ utilizing second order band elimination filter (BEF). The filter consists of a modified step impedance resonator (SIR) and two interdigital coupled line. Two parallel coupled stubs are attached to the SIR to create a second order BEF, which introduce a sharp and wide enough notch. HFSS was used to optimal the structure, and the measured result shown a good agreement with the simulated one. The 3dB bandwidth of the UWB Bandpass filter are between $3.1-4.8 \mathrm{GHz}$ and $6.1-10.2 \mathrm{GHz}$
\end{abstract}

Index Terms - bandpass filter (BPF), notch, parallel coupled stub, ultra-wideband (UWB)

\section{INTRODUCTION}

The unlicensed use of ultra-wideband $(3.1-10.6 \mathrm{GHz})$ frequency spectrum for short range communications was released in 2002 [1]. As an important device of the system, a high performance UWB bandpass is required. Recently, the multiple-mode resonators (MMR) have been applied to UWB filters [2]-[4]. The UWB performance was achieved by using tight coupled of interdigital lines and conventional stepped impedance resonator (SIR), which realize MMR. But a notched bandwidth is needed between $5.15-5.8 \mathrm{GHz}$ to reject the interference from IEEE802.11 wireless network. So many scholars have made great effort on the topic and various kinds of notched techniques were developed. For example, with the embedded open-circuited stub structure for narrow notched band at $5.8 \mathrm{GHz}$ [4], with two asymmetric stubs in the interdigital coupled-line sections a rejection band at 6.6 $\mathrm{GHz}$ was demonstrated [5]. In [6], a small UWB filter using the etched H-shaped slot and the defected ground structure (DGS) was proposed by Yang et al. However, the slots or DGSs etched on the ground plane would destroy the signal integrity for packaging, and all these filters did not guarantee a large suppression between 5.15-5.8GHz.
This paper presents a UWB bandpass filter having suppression larger than $-15 \mathrm{~dB}$ between $5.15-5.8 \mathrm{GHz}$. The filter is constructed by a modified SIR and interdigital coupled-line. Comparing to conventional SIR, the modified one suppress signal between the given bandwidth, while keep the performance same at the other frequency. We get the conception of modified SIR from the conventional parallel coupled line band elimination filter[5]. Firstly a multi-mode resonator UWB bandpass filter is designed, then two open circuited parallel couple line stubs are attached to the SIR to achieve a stop band. Commercial simulator Ansoft HFSS is used to optimized the performance of the filter. The measurement result shows good agreement with the simulation. The filter is fabricated on RT/Duroid 6010 substrate with a thickness of $1.2 \mathrm{~mm}$, permittivity of 10.2 and loss tangent of 0.0023 .

\section{UWB BPF WITH NOTCHED BANDWIDTH}

Fig.1. illustrate the topology of the proposed UWB bandpass filter. It consists of two distinctive parts, i.e., modified SIR and interdigital coupled-lines. As shown in Fig. 1 , the proposed modified SIR is formed by attaching openended parallel coupled stubs at the ends of low impedance transmission line. The stubs alone with the low impedance transmission line form a band elimination filter. The interdigital coupled-line gives a tight coupling from port to the SIR and introduces a transmission zero.

The interdigital coupled line is design first. Length of coupled line is selected as $4.4 \mathrm{~mm}$, which induce a transmission zero at around $14 \mathrm{GHz}$. This transmission zero is aim to suppresses the unwanted fourth resonant mode of SIR filter and make the roll-off at up cut-edge faster. This will be explained in the next section. In order to achieve a tight coupling, the distant of gap is set to $0.1 \mathrm{~mm}$ and the width of coupled line is set to $0.1 \mathrm{~mm}$. The simulation result is shown in Fig. 2.

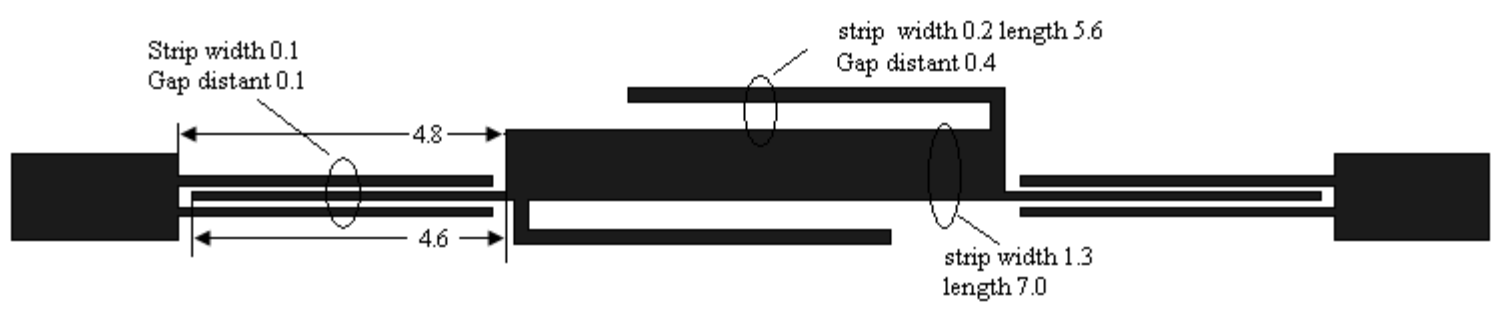

Fig. 1. Proposal notch UWB BPF on microstrip line topology(Unit: millimeter) 
According to papers [2]-[4], when the SIR has an impedance ratio around 2.3, the first three resonant modes are at around 4.23, 6.66 and $9.26 \mathrm{GHz}$, constituting the desired UWB passband. Because the characteristic impedance of $0.1 \mathrm{~mm}$ microstrip line is about $113 \mathrm{Ohm}$, so the low impedance transmission line of SIR is about $49 \mathrm{Ohm}$. Thus the width of low impedance transmission line of SIR is selected as $1.3 \mathrm{~mm}$, corresponding to $490 \mathrm{hm}$. The length of it should be about $1 / 2 \lambda$ ( $\lambda$ is guided wavelength) at $6.9 \mathrm{GHz}$, corresponding to $8.4 \mathrm{~mm}$ [2]. However this value is an approximate one. EM simulation should be used to get the accurate value. We set it to $7.4 \mathrm{~mm}$. The simulation result is shown in Fig. 3, which shows that the first three resonant modes are at around 4.2,6.1 and 9.0GHz. And the fourth resonant mode is at $13.2 \mathrm{GHz}$, which can be suppressed by the interdigital line.

Then a band elimination filter(BEF) is design on the low impedance TML. The stub type BEF ranges the middle band width. In order to achieve a wide enough bandwidth and shape cut-edge, two stubs are attached to SIR to create the second order BEF. Based on the filter theory, the length of stub should be around $1 / 4 \lambda$ ( $\lambda$ is guided wavelength), it means $5.4 \mathrm{~mm}$ at $5.5 \mathrm{GHz}$. So varying the length of stub can tune the location of notch. The distant of gap and width of stub can control the bandwidth of notch and the speed of roll off. Because the length of low impedance TML has only $7.4 \mathrm{~mm}$, it is no enough to design a second order parallel coupled line BEF on it. So the configuration was changed, which is shown in Fig.4 (a). Comparing to the conversional type, the simulation shows that it has a similar frequency response, shown in Fig.4 (b). HFSS was used to optimal the scale of the stubs. Finally length of stub is $5.5 \mathrm{~mm}$, distant of gap is $0.4 \mathrm{~mm}$, stub width is $0.2 \mathrm{~mm}$. The suppression is larger than $-15 \mathrm{~dB}$ between $5.15-6.8 \mathrm{GHz}$, and the $-3 \mathrm{~dB}$ bandwidth is 4.8-6.1GHz.

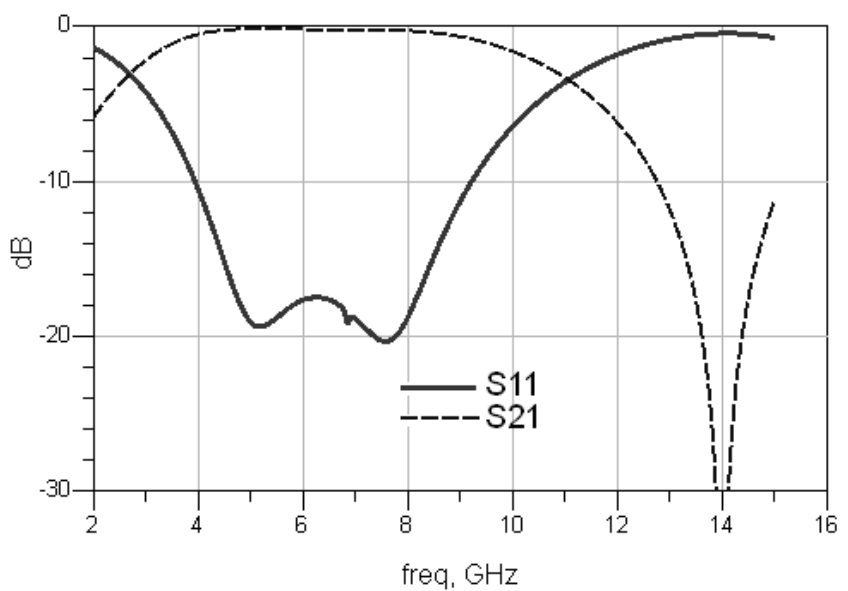

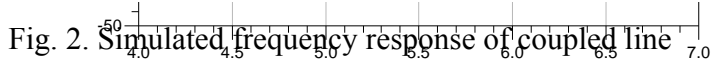
freq, $\mathrm{GHz}$

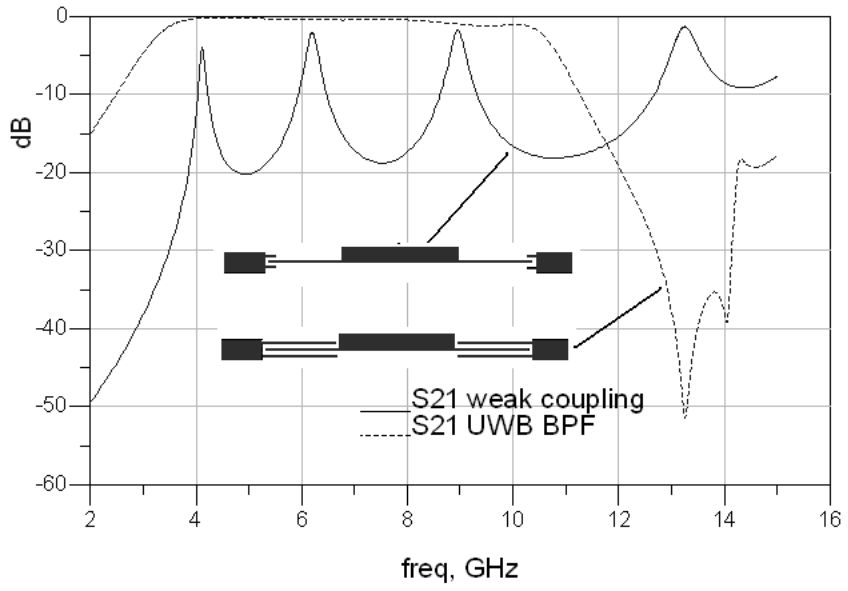

Fig. 3. Simulate frequency response of origin UWB BPF

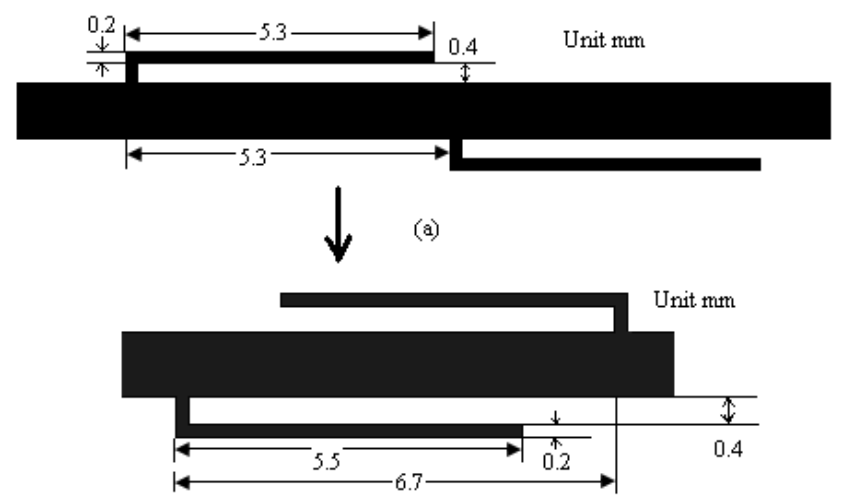

(b)

Fig. 4. topology of second order band elimination filter on microstrip (a) conventional topology (b) modified topology

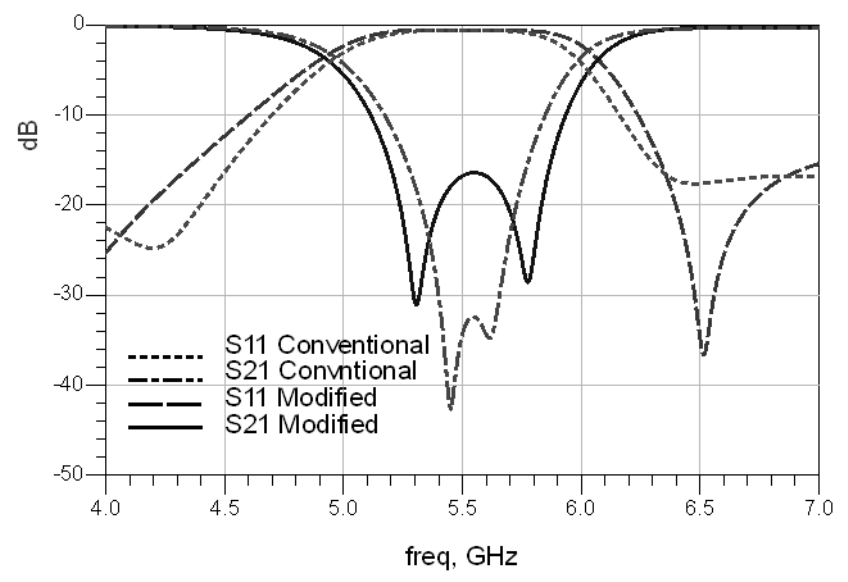

Fig. 5. Simulated S parameters of BEF in Fig. 4. 


\section{SimUlATION AND MEASUREMENT RESUlt}

The UWB bandpass filter is design and fabricate, whose top view photograph is shown in Fig. 6. It was measured by Agilent network analyzer. Fig.7 demonstrates the simulated and measured $|\mathrm{S} 21|$ and $|\mathrm{S} 11|$ in the frequency range of 1 to $15 \mathrm{GHz}$, respectively. It can be seen that the simulated and measured results are in agreement generally. The measured result shows that the $3 \mathrm{~dB}$ bandwidth of the UWB BPF is 3.1$4.8 \mathrm{GHz}$ and $6.1-10.2 \mathrm{GHz}$. The magnitude of S21 is less than $-15 \mathrm{~dB}$ between $5.15 \mathrm{G}-5.8 \mathrm{GHz}$. The simulation result above $11 \mathrm{GHz}$ did not predict the measurement one properly. It may be because of the fabricated or measured error. However, the measured result is still favorable. The size of the fabricated BPF is $16.7 \mathrm{~mm} \times 2.5 \mathrm{~mm}$, i.e., approximately $0.99 \lambda$ by $0.15 \lambda$, $\lambda$ is the wavelength at the center frequency $6.8 \mathrm{GHz}$.

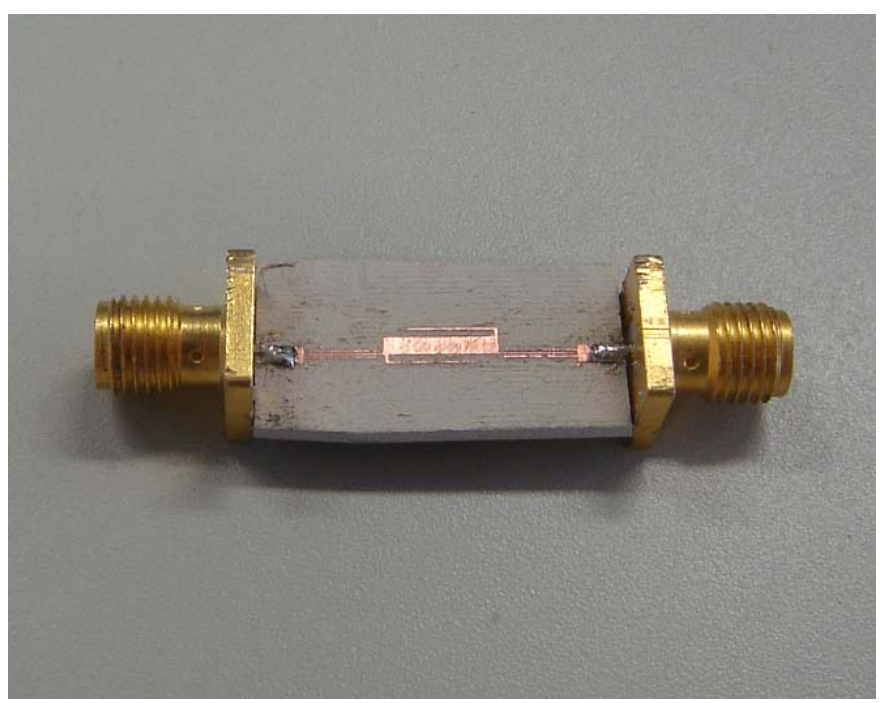

Fig. 6. Top-view photograph of proposal UWB BPF

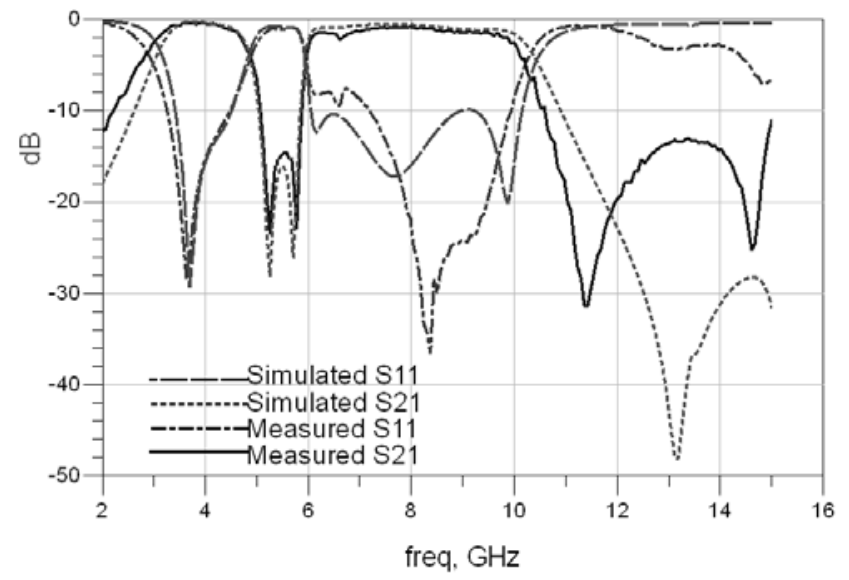

(a)

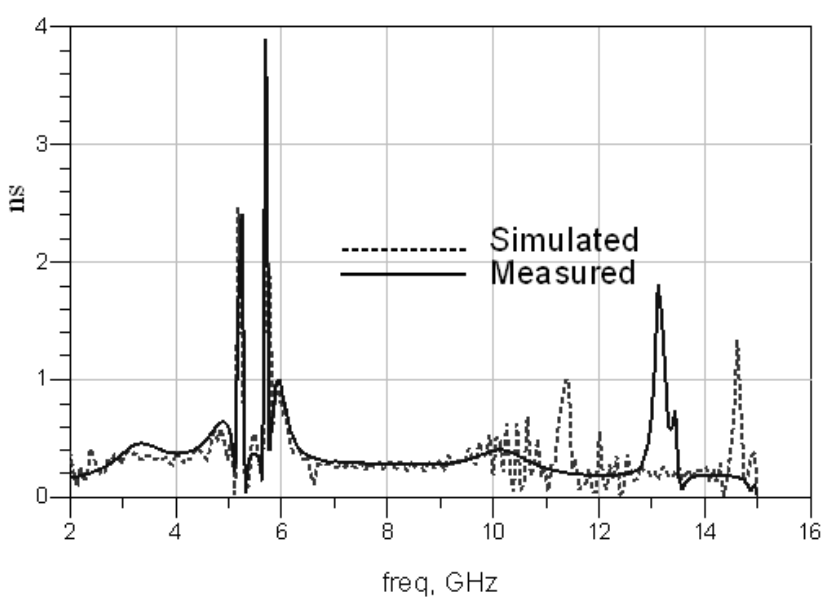

(b)

Fig. 7. Predicted and measured results (a) S- Parameters (b) Group delay

\section{CONCLUSION}

This paper present an high performance and compact UWB bandpass filter based on SIR and interdigital coupled line. With two parallel coupled stubs attached to the SIR, bandstop characteristics larger than $-15 \mathrm{~dB}$ between 5.15 $5.80 \mathrm{GHz}$ is achieved. This filter can be integrated into UWB wireless systems and prohibit the interference from IEEE802.11 devices.

\section{ACKNOWLEDGEMENT}

This work was partly supported by a grant of Knowledge Cluster Initiative implemented by Ministry of Education, Culture, Sports, Science and Technology (MEXT) and JSPS.KAKENHI (Kiban-B).

\section{REFERENCES}

[1] Federal Communications Commission, "Revision of Part 15 of the Commission's Rules Regarding Ultra-Wideband Transmission Systems," Tech. Rep., ET-Docket 98-153, FCC02-48, Apr. 2002

[2] L. Zhu, S. Sun, and W. Menzel, "Ultra-Wideband(UWB) bandpass filters using multiple-mode resonat," IEEE Micro. Wirless Compon. Lett., vol. 15, no.11, pp. 796-798, Nov. 2005.

[3] S. Sun, L. Zhu, "Capactive-ended interdigital coupled lines for UWB bandpass filter with improved out-of-band performances," IEEE Micro. Wirless Compon. Lett., vol. 18, no.3, pp. 173.-175, Mar. 2008.

[4] M. H. Weng, C. T. Liauh, H. W. Wu, "An Ultra-Wideband bandpass filters with an embedded open-circuited stub structure to improve in-band performance," IEEE Micro. Wirless Compon. Lett., vol. 19, no.3, pp. 146-148, Mar. 2009.

[5] H. Shaman, and J. S. Hong, "A novel ultra-wideband (UWB) bandpass filter (BPF) with pairs of transmission zeroes," IEEE 
Micro. Wirless Compon. Lett., vol. 187 no.2, pp. 121-123, Feb. 2007.

[6] G. M. Yang, R. Jin, C. Vittoria, V. G. Harris, and N. X. Sun, "Small Ultra-Wideband(UWB) bandpass filters with notch band ," IEEE Micro. Wirless Compon. Lett., vol. 18, no.3, pp. 176-178, Mar. 2008.

[7] Leo Young, Micowave Filters, Impedance-Matching Networks and Coupling Structures, North Bergen, NJ, USA. Artech House, 1985. 